\title{
Endovascular treatment for emergent large vessel occlusion due to severe intracranial atherosclerotic stenosis
}

\author{
Dong-Hun Kang, MD, ${ }^{1,4}$ Woong Yoon, MD, PhD, ${ }^{2}$ Seul Kee Kim, MD, PhD, ${ }^{2}$ Byung Hyun Baek, MD, ${ }^{2}$ \\ Yun Young Lee, MD, ${ }^{2}$ Yong-Won Kim, MD, ${ }^{3}$ Yong-Sun Kim, MD, PhD, ${ }^{4}$ Yang-Ha Hwang, MD, PhD, ${ }^{3}$ \\ Joon-Tae Kim, MD, PhD, ${ }^{5}$ and Man Seok Park, MD, PhD ${ }^{5}$
}

Departments of ${ }^{1}$ Neurosurgery, ${ }^{4}$ Radiology, and ${ }^{5}$ Neurology, School of Medicine, Kyungpook National University, Daegu; and Departments of ${ }^{2}$ Radiology and ${ }^{3}$ Neurology, Chonnam National University Medical School, Gwangju, Republic of Korea

\begin{abstract}
OBJECTIVE The optimal treatment strategy for patients with emergent large vessel occlusion (ELVO) due to underlying severe intracranial atherosclerotic stenosis (ICAS) is unclear. The purpose of this study was to compare treatment outcomes from intracranial angioplasty with or without stenting and intraarterial infusion of a glycoprotein IIb/IIla inhibitor in patients with ELVO due to severe ICAS, and to investigate predictors of outcome after endovascular therapy in such patients.
\end{abstract}

METHODS A total of 140 consecutive patients with ELVO attributable to severe ICAS underwent endovascular therapy at two stroke centers ( $A$ and $B$ ). Intracranial angioplasty/stenting was primarily performed at center A and intraarterial infusion of glycoprotein Ilb/llla inhibitor (tirofiban) at center B. Data from both centers were prospectively collected into a database and retrospectively analyzed.

RESULTS Overall, successful reperfusion was achieved in 95\% (133/140) of patients and a good outcome in 60\% (84/140). The mortality rate was $7.9 \%$. Symptomatic hemorrhage occurred in 1 patient. There were no significant differences in the rates of successful reperfusion, symptomatic hemorrhage, 3-month modified Rankin scale score 0-2, and mortality between the two centers. Multivariate logistic regression analysis revealed the only independent predictor of good outcome was a history of previous stroke or transient ischemic attack (TIA) (odds ratio 0.254, 95\% confidence interval 0.094-0.689, $p=0.007$ ).

CONCLUSIONS Both intracranial angioplasty/stenting and intraarterial infusion of a glycoprotein IIb/Illa inhibitor are effective and safe in the treatment of underlying severe ICAS in acute stroke patients with ELVO. In addition, a lack of a history of stroke/TIA was the only independent predictor of good outcome after endovascular therapy in such patients.

https://thejns.org/doi/abs/10.3171/2018.1.JNS172350

KEYWORDS atherosclerosis; intracranial; cerebral infarction; thrombectomy; percutaneous transluminal angioplasty; vascular disorders

I NTRACRANIAL atherosclerotic stenosis (ICAS) is one of the most common causes of stroke worldwide and is highly prevalent in black, Asian, and Hispanic populations. $^{4,11}$ The global stroke burden attributed to ICAS is expected to rise because these populations are increasing globally. ${ }^{6,11}$ Thus, it is not uncommon for stroke physicians to encounter acute stroke concomitant with underlying severe ICAS during mechanical thrombectomy for patients with emergent large vessel occlusions (ELVOs), ${ }^{20}$ although the incidence varies from $8.3 \%$ to $60 \%$ according to the geographical area..$^{2,7,15,20}$

Underlying severe ICAS can be a hidden cause of oc-

ABBREVIATIONS DWI = diffusion weighted imaging; ELVO = emergent large vessel occlusion; ICA = internal carotid artery; ICAS = intracranial atherosclerotic stenosis; IQR = interquartile range; IV = intravenous; $\mathrm{MCA}=$ middle cerebral artery; $\mathrm{mRS}=$ modified Rankin scale; $\mathrm{m}$-TICI = modified treatment in cerebral infarction; $\mathrm{NIHSS}=$ National Institutes of Health Stroke Scale; rt-PA = recombinant tissue plasminogen activator; TIA = transient ischemic attack; WASID = Warfarin-Aspirin Symptomatic Intracranial Disease.

SUBMITTED September 19, 2017. ACCEPTED January 15, 2018.

INCLUDE WHEN CITING Published online June 22, 2018; DOI: 10.3171/2018.1.JNS172350. 
clusions refractory to modern mechanical thrombectomy procedures. It should be considered when performing endovascular therapy in patients with ELVO, especially when encountering resistance during mechanical thrombectomy in regions where ICAS is prevalent. ${ }^{15,20}$ Moreover, even if reperfusion is achieved by thrombectomy, immediate re-occlusion occurs frequently in this variant of acute stroke. ${ }^{7,9}$

The optimal treatment strategy for patients with ELVO due to underlying ICAS is unclear. Several case series have reported outcomes after treatment with an intraarterial glycoprotein IIb/IIIa inhibitor or intracranial angioplasty with or without stenting after failed mechanical thrombectomy. ${ }^{2,9,15,20}$ In addition, predictors of outcomes after endovascular stroke therapy in patients with ELVO due to severe ICAS have not been studied. Accordingly, this study aimed to compare treatment outcomes from intracranial angioplasty with or without stenting versus intraarterial infusion of a glycoprotein IIb/IIIa inhibitor in patients with ELVO due to severe ICAS and investigate prognostic factors for a good outcome after endovascular therapy.

\section{Methods \\ Patients}

Between January 2011 and December 2016, a total of 140 consecutive patients with ELVO attributable to underlying severe ICAS $(\geq 70 \%$ stenosis defined by the Warfarin-Aspirin Symptomatic Intracranial Disease [WASID] criteria ${ }^{17}$ ) underwent endovascular therapy at two comprehensive regional stroke centers (A and $\mathrm{B}$ ). Clinical and radiological data from both centers were prospectively collected into a database and retrospectively analyzed. We prospectively collected the following data: demographic features, cerebrovascular risk factors, National Institutes of Health Stroke Scale (NIHSS) score on admission, stroke subtypes according to Trial of Org 10172 in acute stroke treatment (TOAST) classification, ${ }^{1}$ use of intravenous (IV) thrombolysis, time to procedure, procedure time, time to reperfusion, reperfusion status, and 3-month modified Rankin scale (mRS) score. This study was approved by the institutional ethics committee at both centers. Written informed consent for endovascular therapy was obtained from a family member in all patients.

\section{Endovascular Therapy}

All patients underwent a nonenhanced cranial CT scan and multimodal MRI before endovascular therapy. The inclusion criteria for endovascular therapy were as follows: presentation within 8 hours of stroke onset for an anterior circulation stroke and within 12 hours for a posterior circulation stroke; baseline NIHSS score $\geq 4$; no intracranial hemorrhage detected on the cranial CT or MRI; occlusions of the intracranial internal carotid artery (ICA), middle cerebral artery (MCA), or basilar artery detected using MR angiography and confirmed by subsequent catheter angiography; for anterior circulation stroke, infarct volume on diffusion-weighted imaging (DWI) or nonenhanced CT less than one-third of the MCA territory; for posterior circulation stroke, no bi- lateral diffuse pontine ischemia on DWI; and premorbid $\mathrm{mRS}$ score $\leq 3$.

Both cerebral angiography and endovascular therapy were performed under local anesthesia in all patients. When the pretreatment diagnostic angiography showed an intracranial large-vessel occlusion, either stent-retriever or manual aspiration thrombectomy was performed as the first-line endovascular therapy at the discretion of the treating neurointerventionalist. The details of the techniques used for mechanical thrombectomy have been described previously. ${ }^{8,14,18}$ When underlying severe ICAS of the target artery was revealed on the follow-up angiography after a mechanical thrombectomy, two different rescue treatment strategies were used at each stroke center: emergent angioplasty/stenting or intraarterial infusion of tirofiban, a glycoprotein IIb/IIIa inhibitor. ICAS was determined if the fixed stenosis remained unchanged or was aggravated 5-10 minutes after intraarterial injection of a vasodilator through the guide catheter. An angiographic stenotic lesion with an intimal flap or double lumen was considered to be dissection.

At center A, subsequent emergent intracranial angioplasty was performed in all patients (Fig. 1). Subsequent intracranial stenting was performed after angioplasty at the discretion of the neurointerventionalist. The devices and techniques used for intracranial angioplasty and stenting have been described in detail elsewhere. ${ }^{20}$ When instant re-occlusion due to acute thrombosis occurred in the treated artery on the follow-up angiography after angioplasty/stenting, a bolus of 40,000-100,000 IU of urokinase was infused through a microcatheter. Neither heparin nor glycoprotein IIb/IIIa inhibitor was administered either intravenously or intraarterially during the endovascular procedure at center A. Aspirin (160 mg) and clopidogrel $(225 \mathrm{mg}$ ) were given via nasogastric tube immediately after the procedure.

At center $B$, intraarterial infusion of tirofiban was performed as a primary rescue approach in all patients (Fig. 2). Typically, $0.5 \mathrm{mg}$ of tirofiban diluted with $8 \mathrm{ml}$ of normal saline was injected with an infusion pump at a rate of $1 \mathrm{ml} / \mathrm{min}$ through a microcatheter placed just proximal to the stenosis. The total dosage of tirofiban ranged from 0.5 to $1.0 \mathrm{mg}$. A flat-panel detector CT scan (XperCT, Allura Xper FD 20/20; Philips) was performed to exclude intracranial hemorrhage prior to intraarterial tirofiban infusion. Follow-up angiography was performed immediately after and then 10 minutes after completion of infusion. Secondary intracranial angioplasty with stenting was permitted in cases refractory to intraarterial tirofiban infusion, which was defined as no visual improvement in the diameter of the stenotic vessel or blood flow distal to stenosis on follow-up angiography.

\section{Outcome Measures}

The start of endovascular therapy was defined as the moment the needle punctured the common femoral artery. Time to procedure was defined as the interval between time of onset of symptoms and start of endovascular therapy. At both centers, all patients underwent nonenhanced CT scans immediately and 24 hours after endovascular therapy. Intracerebral hemorrhages were assessed on 

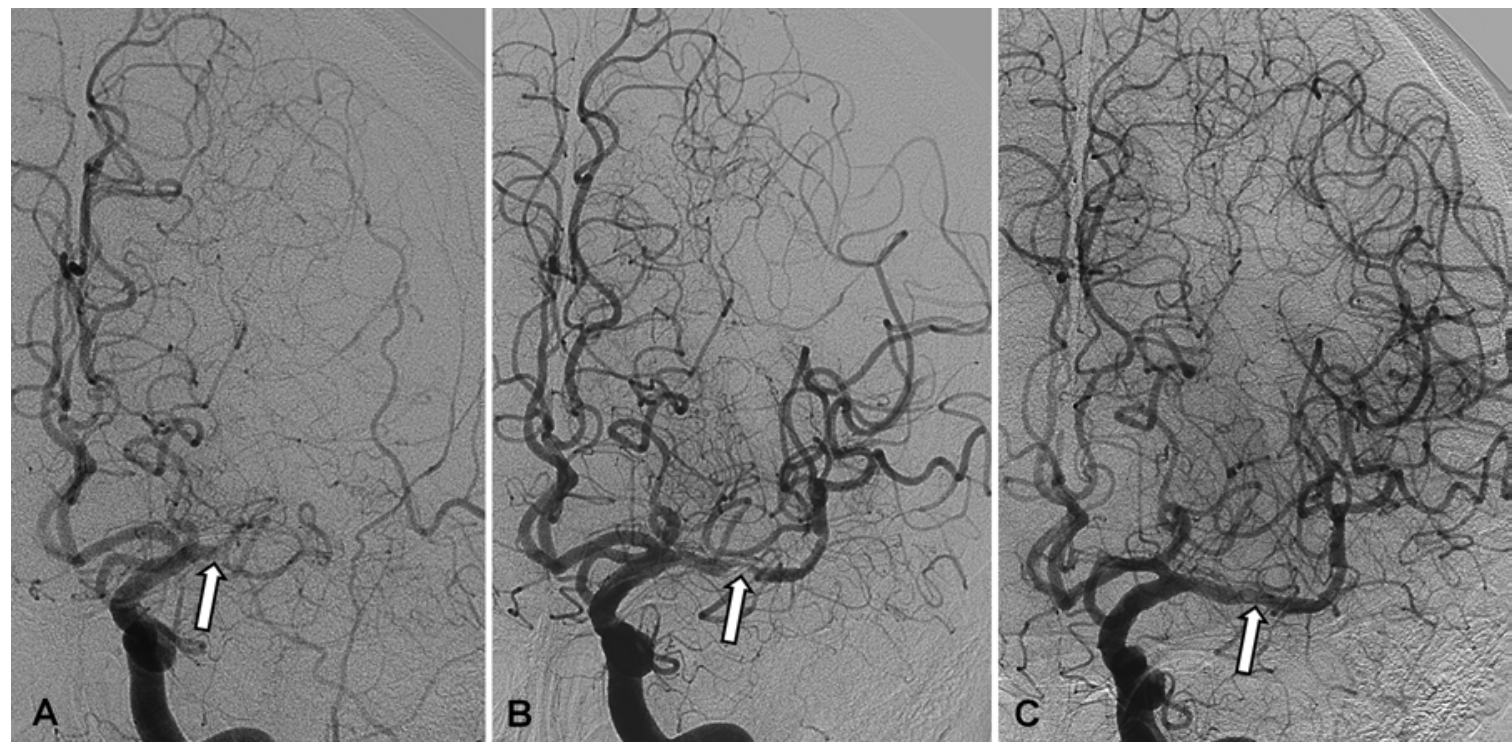

FIG. 1. Brain images from a 73-year-old man with acute ischemic stroke. A: Initial catheter angiography shows the occlusion (arrow) in the proximal $\mathrm{M}_{1}$ segment of the left MCA. B: Angiography obtained after one passage of the Trevo stent-retriever reveals an eccentric severe underlying stenosis (arrow) at the occlusion site. C: Angiography after intracranial angioplasty with a balloon catheter with a diameter of $2.75 \mathrm{~mm}$ shows complete reperfusion in the left MCA territory and mild residual stenosis (arrow).

posttreatment CT scans and classified as hemorrhagic infarction or parenchymal hemorrhage based on the European Cooperative Acute Stroke Study (ECASS) criteria. ${ }^{5}$ Symptomatic intracranial hemorrhage was defined as any intracranial hemorrhage that caused neurological deterioration, defined as a $\geq 4$-point increase in the NIHSS score. Reperfusion status was assessed using the final angiogram according to the modified treatment in cerebral infarction (m-TICI) scale, ${ }^{21}$ with successful reperfusion defined as an m-TICI grade of $2 \mathrm{~b}$ or 3 . Assessment of angiographic images was performed by the consensus of two neuroradiologists (S.K.K. and B.H.B.) who were blinded to the procedure.

Neurological evaluation was performed by a stroke neurologist immediately after treatment and 24 hours and 3 months after treatment, when there were changes in clinical symptoms, and before the patient was discharged. Functional outcome was assessed by a stroke neurologist
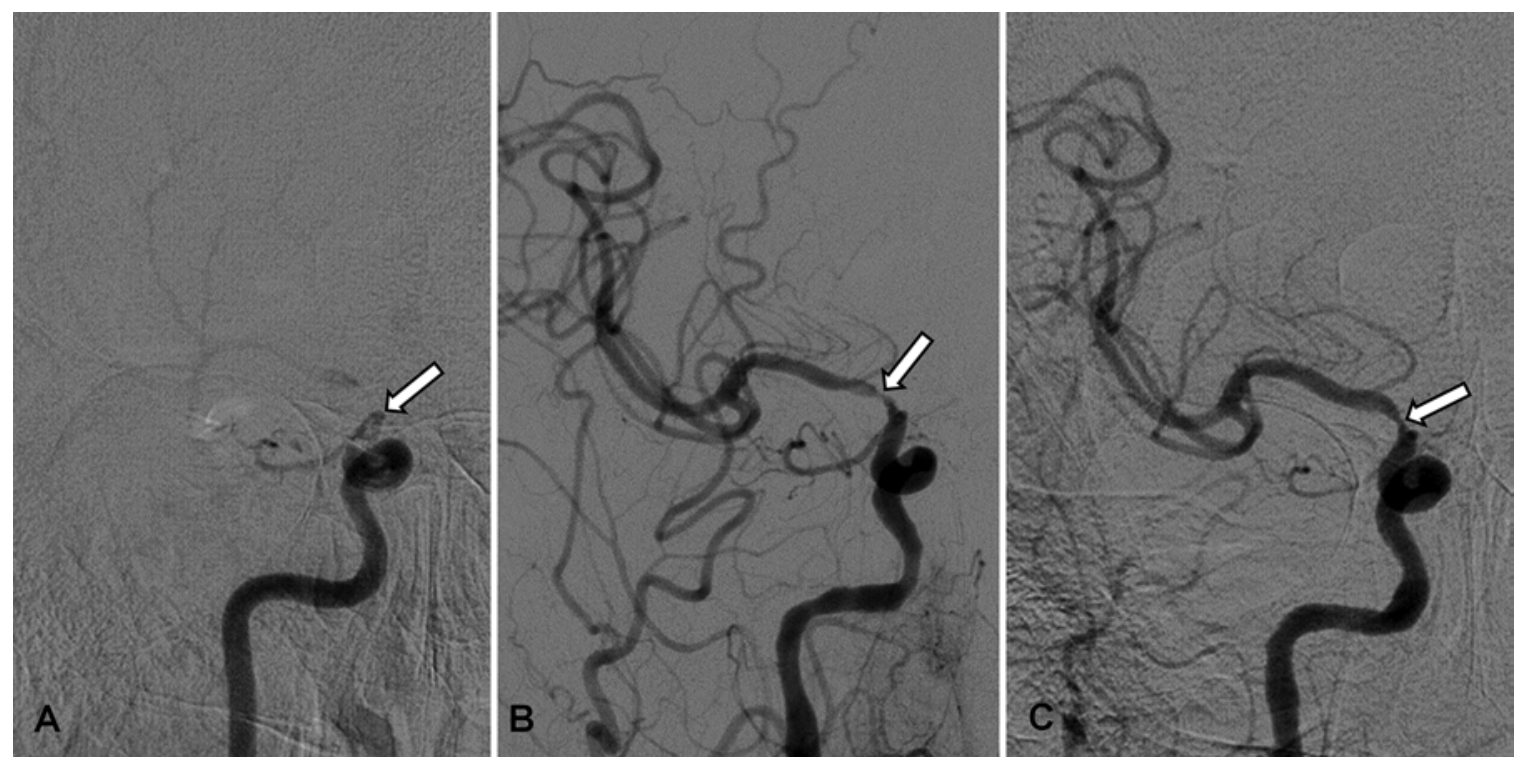

FIG. 2. Brain images from a 69-year-old woman with acute ischemic stroke. A: Initial catheter angiography shows the occlusion (arrow) in the terminal segment of the right ICA. B: Angiography obtained after manual aspiration thrombectomy with a Penumbra reperfusion catheter reveals a concentric severe underlying stenosis (arrow) at the occlusion site. C: Angiography after intraarterial infusion of $0.5 \mathrm{mg}$ of tirofiban through a microcatheter over 10 minutes shows complete reperfusion in the right MCA territory and severe residual stenosis (arrow). This patient has an aplasia of the $A_{1}$ segment of the right anterior cerebral artery. 
TABLE 1. Comparison of baseline characteristics and outcomes of patients from centers $A$ and $B$

\begin{tabular}{|c|c|c|c|}
\hline Variable & Center A $(n=72)$ & Center B $(n=68)$ & $\mathrm{p}$ Value \\
\hline Median age, yrs (IQR) & $66(57-73.75)$ & $68(60.25-75)$ & 0.292 \\
\hline Males, n (\%) & $45(62.5)$ & $46(67.6)$ & 0.523 \\
\hline \multicolumn{4}{|l|}{ Risk factors, n (\%) } \\
\hline Hypertension & $44(61.1)$ & $48(70.6)$ & 0.238 \\
\hline Diabetes mellitus & $23(31.9)$ & $21(30.9)$ & 0.892 \\
\hline Coronary artery disease & $3(4.2)$ & $7(10.3)$ & 0.199 \\
\hline Dyslipidemia & $30(41.7)$ & $29(42.6)$ & 0.907 \\
\hline Smoking & $25(34.7)$ & $35(51.5)$ & 0.060 \\
\hline Atrial fibrillation & $9(12.5)$ & $8(11.8)$ & 0.894 \\
\hline Congestive heart failure & $1(1.4)$ & $3(4.4)$ & 0.356 \\
\hline History of stroke or TIA & $13(18.1)$ & $12(17.6)$ & 0.950 \\
\hline IV rt-PA, n (\%) & $28(38.9)$ & $27(39.7)$ & 0.921 \\
\hline \multicolumn{4}{|l|}{ Site of occlusion, $\mathrm{n}(\%)$} \\
\hline Middle cerebral artery & $41(56.9)$ & $46(67.6)$ & 0.192 \\
\hline Internal carotid artery & $13(18.1)$ & $11(16.2)$ & 0.768 \\
\hline Basilar artery & $18(25)$ & $11(16.2)$ & 0.198 \\
\hline Median time to procedure, $\min (\mathrm{IQR})$ & $240(168.75-357.50)$ & $378.50(176.50-575)$ & 0.001 \\
\hline Median procedure time, min (IQR) & $37.5(30.25-47.75)$ & $53(35.5-83.75)$ & $<0.001$ \\
\hline Median time to reperfusion, $\min (I Q R)$ & $275(205-399.50)$ & $463(274-647.75)$ & $<0.001$ \\
\hline Median baseline NIHSS score (IQR) & $11(9-15)$ & $15(11-20)$ & $<0.001$ \\
\hline Intracranial angioplasty/stenting, n (\%) & $72(100)$ & $6(8.8)$ & $<0.001$ \\
\hline Carotid artery stenting, n (\%) & $1(1.4)$ & $4(5.9)$ & 0.199 \\
\hline $\mathrm{m}-\mathrm{TICl} 2 \mathrm{~b}$ or $3, \mathrm{n}(\%)$ & $69(95.8)$ & $64(94.1)$ & 0.713 \\
\hline Parenchymal hemorrhage, $\mathrm{n}(\%)$ & $3(4.2)$ & $1(1.5)$ & 0.620 \\
\hline Subarachnoid hemorrhage, n (\%) & $7(9.7)$ & $1(1.5)$ & 0.063 \\
\hline Symptomatic hemorrhage, $\mathrm{n}(\%)$ & $1(1.4)$ & 0 & 1 \\
\hline mRS score $0-2$ at 3 mos, $n(\%)$ & $41(56.9)$ & $43(63.2)$ & 0.448 \\
\hline Mortality, n (\%) & $7(9.7)$ & $4(5.9)$ & 0.534 \\
\hline
\end{tabular}

using the mRS score during an outpatient visit 3 months after treatment. If patients were unable to attend the outpatient clinic, the assessment was made via telephone interviews. A good outcome was defined as an mRS score $\leq$ 2 or equal to the premorbid mRS score.

\section{Statistical Analysis}

Continuous variables are presented as medians and interquartile ranges (IQRs). Discrete variables are presented as counts (n) and percentages (\%). The $\chi^{2}$ test or Fisher exact test was used for comparing categorical and binary variables, and the Mann-Whitney U-test was used for comparing continuous variables. First, the baseline characteristics and clinical outcomes of the patients from the two centers (A and B) were compared. Second, the relationship between each clinical and procedural characteristic and the 3-month functional outcome was determined. Third, the independent associations of the 3-month functional outcome with other clinical and radiological variables were determined using a multivariate logistic regression analysis. The variables tested in the logistic regression models were those with $p$ values $<0.1$ in the univariate analysis. All statistical analyses were performed with SPSS software (version 23.0, IBM SPSS). A p value $<0.05$ was considered significant.

\section{Results}

The data from 140 patients (91 men and 49 women, median age 67 years, range 30-88 years) were analyzed (Table 1). Center A included 72 patients and center B included 68. During the study period, the proportion of ICAS among patients who received mechanical thrombectomy for ELVO was $14.2 \%$ (72/508) in center A and $15.2 \%(68 / 447)$ in center B. Underlying severe ICAS that was attributed to ELVO and acute ischemic symptoms was most frequently observed in the MCA $(n=87)$ followed by the basilar artery $(n=29)$ and the ICA $(n=24)$. In the entire cohort, $65.7 \%(92 / 140)$ of patients had hypertension, $42.9 \%(60 / 140)$ had a smoking history, $42.1 \%(59 / 140)$ had dyslipidemia, 31.4\% (44/140) had diabetes mellitus, $17.9 \%$ $(25 / 140)$ had a history of prior ischemic stroke or transient ischemic attack (TIA), 12.1\% (17/140) had atrial fibrillation, $7.1 \%(10 / 140)$ had a history of previous coronary artery disease, and 2.9\% (4/140) had congestive heart failure. The median NIHSS score on admission was 13. Intra- 
TABLE 2. Comparison between patients with good outcome and poor outcome after endovascular therapy for ELVO due to severe ICAS

\begin{tabular}{|c|c|c|c|}
\hline Variable & Good Outcome $(n=84)$ & Poor Outcome $(n=56)$ & $p$ Value \\
\hline Median age, yrs (IQR) & $65.5(55.25-72.75)$ & $70(61.25-77.75)$ & 0.006 \\
\hline Males, n (\%) & $60(71.4)$ & $31(55.4)$ & 0.051 \\
\hline \multicolumn{4}{|l|}{ Risk factors, n (\%) } \\
\hline Hypertension & $58(69.0)$ & $34(60.7)$ & 0.309 \\
\hline Diabetes mellitus & $28(33.3)$ & $16(28.6)$ & 0.552 \\
\hline Coronary artery disease & $3(3.6)$ & $7(12.5)$ & 0.089 \\
\hline Dyslipidemia & $38(45.2)$ & $21(37.5)$ & 0.364 \\
\hline Smoking & $41(48.8)$ & $19(33.9)$ & 0.081 \\
\hline Atrial fibrillation & $8(9.5)$ & $9(16.1)$ & 0.245 \\
\hline Congestive heart failure & $2(2.4)$ & $2(3.6)$ & 1 \\
\hline History of stroke or TIA & $7(8.3)$ & $18(32.1)$ & $<0.001$ \\
\hline IV rt-PA, n (\%) & $37(44.0)$ & $18(32.1)$ & 0.158 \\
\hline \multicolumn{4}{|l|}{ Site of occlusion, n (\%) } \\
\hline Middle cerebral artery & $55(65.5)$ & $32(57.1)$ & 0.319 \\
\hline Internal carotid artery & $15(17.9)$ & $9(16.1)$ & 0.784 \\
\hline Basilar artery & $14(16.7)$ & $15(26.8)$ & 0.148 \\
\hline Median time to procedure, $\min (\mathrm{IQR})$ & $262.5(162.25-430)$ & $327.5(224-409)$ & 0.204 \\
\hline Median procedure time, $\min (\mathrm{IQR})$ & $41(28-59.75)$ & $45(33.25-65)$ & 0.144 \\
\hline Median time to reperfusion, $\min (\mathrm{IQR})$ & $301(221.25-508)$ & $392.5(275-481.25)$ & 0.112 \\
\hline Median baseline NIHSS score (IQR) & $12.5(9-17)$ & $14.5(10-20)$ & 0.056 \\
\hline Intracranial angioplasty/stenting, n (\%) & $44(52.4)$ & $34(60.7)$ & 0.331 \\
\hline Carotid artery stenting, $\mathrm{n}(\%)$ & $3(3.6)$ & $2(3.6)$ & 1 \\
\hline $\mathrm{m}-\mathrm{TICl} 2 \mathrm{~b}$ or $3, \mathrm{n}(\%)$ & $81(96.4)$ & $52(92.9)$ & 0.437 \\
\hline Parenchymal hemorrhage, n (\%) & $2(2.4)$ & $2(3.6)$ & 1 \\
\hline Subarachnoid hemorrhage, $n(\%)$ & $4(4.8)$ & $4(7.2)$ & 0.713 \\
\hline Symptomatic hemorrhage, n (\%) & 0 & $1(1.8)$ & 0.4 \\
\hline
\end{tabular}

venous thrombolysis was performed prior to endovascular therapy in $39.3 \%(55 / 140)$ of the patients.

Overall, successful reperfusion (m-TICI $2 \mathrm{~b}$ or 3) was achieved in $95 \%(133 / 140)$ of patients and a good outcome (mRS score 0-2) in 60\% (84/140). Parenchymal hemorrhage occurred in $2.9 \%(4 / 140)$ and subarachnoid hemorrhage in $5.7 \%$ (8/140). Symptomatic hemorrhage occurred in 1 patient. The mortality rate was $7.9 \%(11 / 140)$.

\section{Comparisons of Baseline Characteristics and Outcomes Between Centers A and B}

The results of the comparison of patients from center $A$ versus center B are shown in Table 1. There were no significant differences between the patients from the two centers in age, sex, cerebrovascular risk factors, occlusion sites, and the use of IV recombinant tissue plasminogen activator (rt-PA). The baseline NIHSS score was higher in center B patients than center A (11 vs 15, p <0.001). The median time to procedure (240 vs $378.5 \mathrm{~min}, \mathrm{p}<0.001$ ), procedure time $(37.5$ vs $53 \mathrm{~min}, \mathrm{p}<0.001)$, and time to reperfusion (275 vs $463 \mathrm{~min}, \mathrm{p}<0.001$ ) were significantly longer in center B patients than in center A. Stent-retriever thrombectomy was exclusively used as the first-line mechanical thrombectomy in center $\mathrm{A}$ and manual aspiration thrombectomy was used predominantly in center B
(80.9\%, $\mathrm{n}=55 / 68)$. Intracranial angioplasty/stenting was performed more frequently in center $\mathrm{A}$ than center $\mathrm{B}$ $(100 \%$ vs $8.8 \%, \mathrm{p}<0.001)$. Of the 72 patients in center $\mathrm{A}$, $46(64 \%)$ underwent angioplasty with stenting and 26 were treated with angioplasty alone. All 6 patients in center B underwent angioplasty with stenting. Subarachnoid hemorrhage tended to occur more frequently in center A patients than center B $(9.7 \%$ vs $1.5 \%, \mathrm{p}=0.063)$. There were no significant differences between the two centers in the rate of successful reperfusion, parenchymal hemorrhage, symptomatic hemorrhage, 3-month mRS score, and mortality rate. There was no vessel rupture or angiographic extravasation of contrast medium during procedures in either center. In center A, 4 patients (5.6\%) received intraarterial urokinase infusion due to instant re-occlusion after emergent angioplasty/stenting. These 4 patients achieved successful revascularization on final angiograms. In center $\mathrm{B}, 6$ patients $(8.8 \%)$ received rescue angioplasty and stenting due to instant re-occlusion. Successful revascularization was achieved in 4 of these 6 patients.

\section{Associations Between Clinical and Procedural Factors and Functional Outcome}

The results of the comparison between the patients with a good outcome and those with a poor outcome are shown 
in Table 2. Patients with a good outcome were younger (65.5 years vs 70 years, $p=0.006$ ) and had a less frequent history of a previous stroke or TIA $(8.3 \%$ vs $32.1 \%$, p < $0.001)$. There were also trends toward male predominance (71.4\% vs $55.4 \%, \mathrm{p}=0.051)$ and lower baseline NIHSS score $(12.5$ vs $14.5, \mathrm{p}=0.056)$ in patients with a good outcome.

Multivariate logistic regression analysis, including variables with a $\mathrm{p}$ value $<0.1$ in a univariate analysis (age, sex, baseline NIHSS, history of previous coronary artery disease, history of previous stroke/TIA, and history of smoking), revealed the only independent predictor of good functional outcome was a lack of history of previous stroke or TIA (odds ratio $=0.254,95 \%$ confidence interval 0.094-0.689, $\mathrm{p}=0.007)$.

\section{Discussion}

The main findings of the present study were: 1) modern endovascular therapy can achieve high rates of successful reperfusion, good functional outcome, and a low rate of mortality in patients with acute ischemic stroke attributable to underlying severe ICAS; 2) there were no differences in treatment outcomes between patients treated with emergent angioplasty/stenting versus intraarterial infusion of a glycoprotein IIb/IIIa inhibitor; and 3) a lack of a history of previous stroke or TIA was the only independent predictor of good functional outcome after endovascular therapy in this patient population.

\section{Treatment Options for ELVO Due to ICAS}

Although the effectiveness of endovascular therapy in patients with acute ischemic stroke due to ELVO has now been demonstrated in recent randomized controlled studies, most of these patients had embolic occlusions. The optimal treatment for ELVO with underlying ICAS remains to be elucidated and there have been very few studies conducted on this topic. . $^{2,15,20}$ Currently, there are two potential endovascular therapeutic options when encountering underlying ICAS during mechanical thrombectomy procedures in patients with ELVO: angioplasty with or without stenting, and intraarterial infusion of an antiplatelet drug.

The major components of clots formed by in situ thrombosis are platelets and fibrin. ${ }^{12,13}$ Thus, local infusion of an antiplatelet drug, such as a glycoprotein IIb/ IIIa inhibitor at the occlusion site, may be a reasonable therapeutic option in such patients. Tirofiban is a shortacting glycoprotein IIb/IIIa inhibitor and competitively inhibits platelet aggregation mediated by fibrinogen. The intraarterial use of tirofiban during neurointerventional procedures is known to be effective and safe. ${ }^{3,10}$ The main disadvantage of this approach would be instant or early re-occlusion of the treated artery because the severe stenosis itself remains unsolved., 9 Recent studies also suggested that emergent angioplasty with or without stenting can be a viable therapeutic option in patients with acute stroke secondary to underlying ICAS. Yoon et al. reported that emergent angioplasty/stenting was associated with a high rate of good outcome and a low rate of hemorrhagic complications in such patients. ${ }^{20}$ Expected disadvantages of intracranial angioplasty/stenting in the setting of acute stroke include vessel injury and in-stent thrombosis. To our knowledge, this is the first study to compare these two therapeutic options in patients with acute stroke secondary to underlying ICAS. Our study demonstrates that both treatment approaches are equally safe and effective in the treatment of ELVO due to severe ICAS. In our study, overall successful reperfusion was achieved in $95 \%$ and good functional outcome in $60 \%$ of patients. There was one symptomatic hemorrhage and mortality was only $7.9 \%$ at 3 months. Based on the results of our study, we suggest that treating physicians may choose the therapeutic option they are familiar with, either intracranial angioplasty/ stenting or intraarterial infusion of a glycoprotein IIb/IIIa inhibitor, as an adjuvant strategy for treating patients with ELVO due to severe ICAS. If one of either of the methods failed to achieve effective reperfusion, the other can be used as a rescue approach. Further multicenter randomized controlled studies are still needed to establish the optimal treatment for ELVO secondary to severe ICAS.

An alternative treatment option for ELVO with severe ICAS is just to perform a mechanical thrombectomy alone and best medical therapy following thrombectomy. It is unclear at this moment that any additional endovascular procedures are required after adequate reperfusion has been achieved by thrombectomy, even if there is an underlying stenosis. These patients have presumably had long-standing stenosis that has not been symptomatic until the time of the acute event. These patients could be placed on intensive medical therapy after thrombectomy, which would include antiplatelet medication.

\section{Predictors of Outcomes in Patients With ELVO Due to ICAS}

There have been no reports regarding predictors of outcome after endovascular therapy in patients with ELVO and severe ICAS. Our study suggests that patients with recurrent stroke due to severe ICAS are likely to have poor functional outcome if they are presenting with ELVO. In the present study, we identified a lack of a history of previous stroke or TIA as the only independent predictor of good outcome (odds ratio 0.254, $\mathrm{p}=0.007$ ) after endovascular therapy in patients with ELVO due to severe ICAS. The WASID trial suggested that patients with symptomatic ICAS $\geq 70 \%$ are at high risk of subsequent stroke, predominantly in the territory of the stenotic artery. ${ }^{11}$ Thus, our results together with the WASID trial reemphasize that more meticulous management of vascular risk factors is needed to prevent secondary stroke in patients with firstever stroke and severe intracranial stenosis $\geq 70 \%$.

Our study confirms that achieving effective, quality reperfusion is one of the most important determinants of functional outcomes and quality reperfusion is more important than time in patients with acute stroke undergoing endovascular therapy, even in this variant of acute stroke due to underlying severe ICAS. ${ }^{16,19}$ When comparing two centers in the present analysis, there was significant baseline imbalance in the initial NIHSS score, time to procedure, procedure time, and time to reperfusion between the two centers. However, there were no significant differences in the rates of 3-month good outcome and mortality. This 
was probably due to the high rate of successful reperfusion achieved in both centers (95.8\% and $94.1 \%$, respectively). Moreover, the safety parameters, including the rates of parenchymal hemorrhage and symptomatic hemorrhage, were not significantly different between centers.

\section{Limitations of the Study}

This study has several limitations, including its retrospective and noncontrolled study design. In addition, angiographic stenosis after mechanical thrombectomy might occur due to residual adherent clot, dissection, or vasospasm; these would be difficult to differentiate from an atherosclerotic stenosis. However, fragmented residual clots would migrate to distal branches and do not adhere to the vessel wall when antegrade blood flow is restored after mechanical thrombectomy. Arterial dissection could be easily differentiated by its characteristic angiographic appearance, such as an intimal flap or double lumen. We performed additional angiographic runs 5-10 minutes after intraarterial injection of a vasodilator to differentiate fixed stenosis from vasospasm. Finally, there were baseline differences between the patients from the two centers in terms of initial NIHSS score, time to procedure, procedure time, and time to reperfusion, which might affect the results of this study. However, as mentioned earlier, these differences emphasize the importance of successful reperfusion in achieving good functional outcome in this patient population.

\section{Conclusions}

This study is the largest study showing that modern endovascular therapy can achieve a high rate of successful reperfusion and good outcome as well as a low rate of complications and mortality in acute stroke patients with ELVO due to severe ICAS. Our study suggests that both intracranial angioplasty/stenting and intraarterial infusion of a glycoprotein IIb/IIIa inhibitor are effective and safe in the treatment of underlying severe ICAS in patients with acute ischemic stroke. Further multicenter randomized controlled studies are still needed to determine the optimal treatment strategy in such patients. This treatment strategy should also include thrombectomy alone and subsequent best medical therapy. In addition, we found that a previous history of stroke/TIA was associated with a poor functional outcome at 3 months after endovascular therapy in this patient population. Thus, more meticulous management of vascular risk factors is needed to prevent secondary stroke in patients with first-ever stroke and severe ICAS.

\section{Acknowledgments}

This study was supported by a grant (no. CRI 15901-22.1) from Chonnam National University Hospital Biomedical Research Institute.

\section{References}

1. Adams HP Jr, Bendixen BH, Kappelle LJ, Biller J, Love $\mathrm{BB}$, Gordon DL, et al: Classification of subtype of acute ischemic stroke. Definitions for use in a multicenter clinical trial. TOAST. Trial of Org 10172 in Acute Stroke Treatment. Stroke 24:35-41, 1993
2. Al Kasab S, Almadidy Z, Spiotta AM, Turk AS, Chaudry MI, Hungerford JP, et al: Endovascular treatment for AIS with underlying ICAD. J Neurointerv Surg 9:948-951, 2017

3. Baik SK, Oh SJ, Park KP, Lee JH: Intra-arterial tirofiban infusion for partial recanalization with stagnant flow in hyperacute cerebral ischemic stroke. Interv Neuroradiol 17:442-451, 2011

4. Banerjee C, Chimowitz MI: Stroke caused by atherosclerosis of the major intracranial arteries. Circ Res 120:502-513, 2017

5. Fiorelli M, Bastianello S, von Kummer R, del Zoppo GJ, Larrue V, Lesaffre E, et al: Hemorrhagic transformation within 36 hours of a cerebral infarct: relationships with early clinical deterioration and 3-month outcome in the European Cooperative Acute Stroke Study I (ECASS I) cohort. Stroke 30:2280-2284, 1999

6. Gorelick PB, Wong KS, Bae HJ, Pandey DK: Large artery intracranial occlusive disease: a large worldwide burden but a relatively neglected frontier. Stroke 39:2396-2399, 2008

7. Hwang YH, Kim YW, Kang DH, Kim YS, Liebeskind DS: Impact of target arterial residual stenosis on outcome after endovascular revascularization. Stroke 47:1850-1857, 2016

8. Kang DH, Hwang YH, Kim YS, Park J, Kwon O, Jung C: Direct thrombus retrieval using the reperfusion catheter of the penumbra system: forced-suction thrombectomy in acute ischemic stroke. AJNR Am J Neuroradiol 32:283-287, 2011

9. Kang DH, Kim YW, Hwang YH, Park SP, Kim YS, Baik SK: Instant reocclusion following mechanical thrombectomy of in situ thromboocclusion and the role of low-dose intra-arterial tirofiban. Cerebrovasc Dis 37:350-355, 2014

10. Kang HS, Kwon BJ, Roh HG, Yoon SW, Chang HW, Kim JE, et al: Intra-arterial tirofiban infusion for thromboembolism during endovascular treatment of intracranial aneurysms. Neurosurgery 63:230-238, 2008

11. Kasner SE, Chimowitz MI, Lynn MJ, Howlett-Smith H, Stern BJ, Hertzberg VS, et al: Predictors of ischemic stroke in the territory of a symptomatic intracranial arterial stenosis. Circulation 113:555-563, 2006

12. Kim SK, Yoon W, Heo TW, Park MS, Kang HK: Negative susceptibility vessel sign and underlying intracranial atherosclerotic stenosis in acute middle cerebral artery occlusion. AJNR Am J Neuroradiol 36:1266-1271, 2015

13. Kim SK, Yoon W, Kim TS, Kim HS, Heo TW, Park MS: Histologic analysis of retrieved clots in acute ischemic stroke: correlation with stroke etiology and gradient-echo MRI. AJNR Am J Neuroradiol 36:1756-1762, 2015

14. Kim SK, Yoon W, Moon SM, Park MS, Jeong GW, Kang HK: Outcomes of manual aspiration thrombectomy for acute ischemic stroke refractory to stent-based thrombectomy. J Neurointerv Surg 7:473-477, 2015

15. Lee YY, Yoon W, Kim SK, Baek BH, Kim GS, Kim JT, et al: Acute basilar artery occlusion: differences in characteristics and outcomes after endovascular therapy between patients with and without underlying severe atherosclerotic stenosis. AJNR Am J Neuroradiol 38:1600-1604, 2017

16. Manning NW, Chapot R, Meyers PM: Endovascular stroke management: key elements of success. Cerebrovasc Dis 42:170-177, 2016

17. Samuels OB, Joseph GJ, Lynn MJ, Smith HA, Chimowitz MI: A standardized method for measuring intracranial arterial stenosis. AJNR Am J Neuroradiol 21:643-646, 2000

18. Yoon W, Jung MY, Jung SH, Park MS, Kim JT, Kang HK: Subarachnoid hemorrhage in a multimodal approach heavily weighted toward mechanical thrombectomy with solitaire stent in acute stroke. Stroke 44:414-419, 2013

19. Yoon W, Kim SK, Park MS, Baek BH, Lee YY: Predictive factors for good outcome and mortality after stent-retriever 
thrombectomy in patients with acute anterior circulation stroke. J Stroke 19:97-103, 2017

20. Yoon W, Kim SK, Park MS, Kim BC, Kang HK:

Endovascular treatment and the outcomes of atherosclerotic intracranial stenosis in patients with hyperacute stroke. Neurosurgery 76:680-686, 2015

21. Zaidat OO, Yoo AJ, Khatri P, Tomsick TA, von Kummer $\mathrm{R}$, Saver JL, et al: Recommendations on angiographic revascularization grading standards for acute ischemic stroke: a consensus statement. Stroke 44:2650-2663, 2013

\section{Disclosures}

The authors report no conflict of interest concerning the materials or methods used in this study or the findings specified in this paper.

\section{Author Contributions}

Conception and design: Yoon, Kang. Acquisition of data: Yoon, Kang, SK Kim, Baek, Lee, YW Kim, Hwang, JT Kim, Park. Analysis and interpretation of data: Yoon, Kang, YS Kim, Park. Drafting the article: Yoon, Kang. Critically revising the article: Yoon. Reviewed submitted version of manuscript: Yoon. Approved the final version of the manuscript on behalf of all authors: Yoon. Statistical analysis: Yoon. Study supervision: Yoon.

\section{Correspondence}

Woong Yoon: Chonnam National University Medical School, Chonnam National University Hospital, Gwangju, Republic of Korea.radyoon@jnu.ac.kr. 